\title{
Compact Silicon Nitride Arrayed Waveguide Gratings for Very Near-Infrared Wavelengths
}

\author{
Daan Martens, Ananth Z. Subramanian, Shibnath Pathak, Michael Vanslembrouck, \\ Peter Bienstman, Wim Bogaerts, and Roel G. Baets
}

\begin{abstract}
In this letter, we report a novel high-index-contrast silicon nitride arrayed waveguide grating (AWG) for very nearinfrared wavelengths. This device is fabricated through a process compatible with a complementary metal-oxide-semiconductor fabrication line and is therefore suitable for mass fabrication. The large phase errors that usually accompany high-index-platform AWGs are partly mitigated through design and fabrication adaptions, in particular the implementation of a two-level etch scheme. Multiple devices are reported, among which a $0.3-\mathrm{mm}^{2}$ device which, after the subtraction of waveguides loss, has a $-1.2 \mathrm{~dB}$ on-chip insertion loss at the peak of the central channel and 20-dB crosstalk for operation $\sim 900 \mathrm{~nm}$ with a channel spacing of $2 \mathrm{~nm}$. These AWGs pave the way for numerous largescale on-chip applications pertaining to spectroscopy and sensing.
\end{abstract}

Index Terms-Near-infrared, arrayed waveguide grating, silicon nitride, high-index-contrast.

\section{INTRODUCTION}

A RRAYED waveguide gratings (AWGs) are an essential building block for integrated photonic systems due to their compactness and excellent performance [1]. Their main fiel of application is wavelength-division multiplexing for optical communications [2]. Additionally, they are increasingly being used in lab-on-a-chip based systems for photonic sensing like spectroscopy [3] and refractive index sensing [4]. Due to this versatility, designs have been made for various material platforms and wavelength ranges [5]-[7]. AWGs for the nearinfrared and visible range have also been developed [8]-[12]. This very near-infrared (VNIR) wavelength region $(700-1000 \mathrm{~nm})$ is of particular interest due to its negligible water absorption, low fuorescence, the availability of highquality low-cost sources and detectors as well as the presence of the therapeutic window for minimal photo damage to the

Manuscript received June 20, 2014; revised October 7, 2014; accepted October 9, 2014. Date of publication October 17, 2014; date of current version December 17, 2014. This work was carried out in the framework of ERCInSpectra Advanced Grant. The work of D. Martens and P. Bienstman was supported by the EU FP7 Pocket project.

D. Martens, A. Z. Subramanian, S. Pathak, M. Vanslembrouck, P. Bienstman, and R. G. Baets are with the Center for NanoBiophotonics, Ghent University-imec, Ghent 9000, Belgium (e-mail: daan.martens@intec.ugent.be; ananth.subramanian@intec.ugent.be; shibnath.pathak@intec.ugent.be; michael.vanslembrouck@intec.ugent.be; peter.bienstman@ugent.be; roel.baets@intec.ugent.be).

W. Bogaerts is with the Center for Nano-Biophotonics, Ghent University-imec, Ghent 9000, Belgium, and also with Luceda Photonics, Dendermonde 9200, Belgium (e-mail: wim.bogaerts@ugent.be).

Color versions of one or more of the fgures in this letter are available online at $\mathrm{http}: / /$ ieeexplore.ieee.org.

Digital Object Identifie 10.1109/LPT.2014.2363298 tissues, making it ideal for sensing applications. The current devices in this wavelength region are however restricted to large footprints due to their low-index-contrast materials. In a high-index-contrast (HIC) material platform on the other hand, very sharp bends are possible and devices can therefore be made more compact. This footprint reduction allows integrating much more components on a single chip. The most mature high-index-contrast system is silicon-oninsulator, for example a 16-channel $0.41 \mathrm{~mm}^{2}$ AWG with $-1.6 \mathrm{~dB}$ insertion loss and conventional crosstalk of $22.5 \mathrm{~dB}$ has been reported at $1550 \mathrm{~nm}$ [13]. Unfortunately, silicon-oninsulator lacks transparency below $1.1 \mu \mathrm{m}$ and can therefore not be used in the VNIR wavelength region. Silicon nitride $\left(\mathrm{Si}_{3} \mathrm{~N}_{4}\right)$ is a transparent alternative, conserving compatibility with a complementary metal-oxide-semiconductor (CMOS) fabrication line and possessing a relatively high refractive index of around 1.9 in the VNIR wavelength regime. In this platform, AWGs have already been demonstrated operating in the telecom wavelength region [14]-[16], but in this letter devices for shorter wavelengths are described. Recently, we reported single-mode low-loss $\mathrm{Si}_{3} \mathrm{~N}_{4}$ wire waveguides for visible VNIR wavelengths with losses $<1 \mathrm{~dB} / \mathrm{cm}$ at $900 \mathrm{~nm}$ [17]. HIC AWGs typically are more sensitive to sidewall roughness, causing high phase errors. This sensitivity reduces the margin of error on design and fabrication [13]. Including a shallow etch allows decreasing the index contrast locally at critical parts and thereby improving the device performance [2]. This letter presents the results of a novel $\mathrm{Si}_{3} \mathrm{~N}_{4}$ AWG, operating in the $900 \mathrm{~nm}$ wavelength range. These devices combine a very small footprint with low insertion losses, making them suitable for diverse on-chip applications.

\section{AWG THEORY}

AWGs are feed-forward spectral flters, combining a multipath delay with two free-space focusing regions, referred to as star couplers, as illustrated in Fig. 1(a). Light entering the input star coupler diverges and gets coupled into an array of waveguides. Their length difference $\Delta \mathrm{L}$ is such that their optical path length difference equals an integral multiple of the central wavelength, $\Delta \mathrm{L}=\mathrm{m} \lambda_{c} / \mathrm{n}_{\text {eff. }}$. When diverging from $\lambda_{c}$, the outgoing beam will be shifted along the image plane. The receiver waveguides are placed along this image plane, each capturing a different section of the spatially separated spectrum. More information on AWG theory can be found in [1]. 

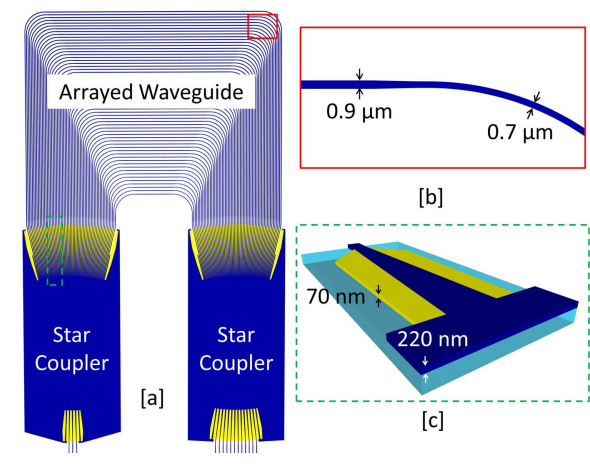

[b]

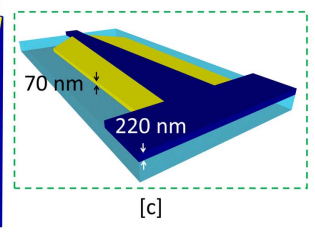

Fig. 1. (a) Top view of silicon nitride AWG. (b) Detail of waveguide array. (c) Shallow etch aperture of star coupler.

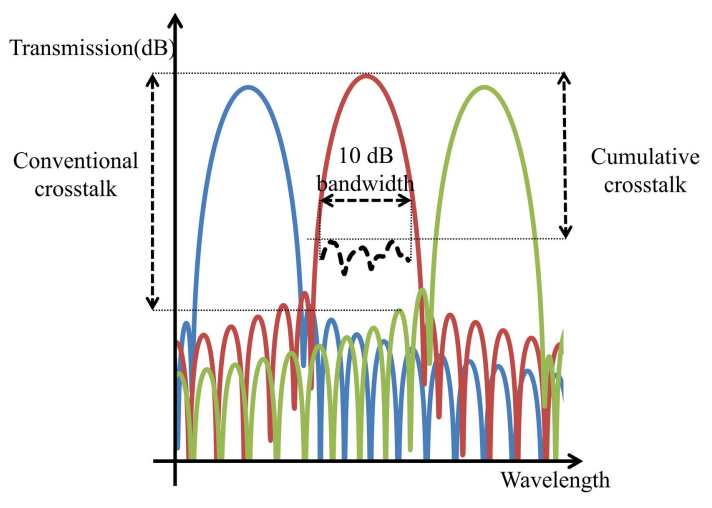

Fig. 2. Def nition of both types of crosstalk of a channel of an AWG spectrum. The dotted curve is the total power of all other channels within the wavelength $10 \mathrm{~dB}$-band of the central channel.

Typical parameters to characterize such a device are peak loss of the central channel (referred to as insertion loss), its difference with the peak loss of the lowest, usually outer channels (known as insertion loss non-uniformity) and the crosstalk. For describing this crosstalk, two f gures of merit will be applied: First, the conventional crosstalk, def ned per channel as the difference between the power at peak wavelength and the peak power of any other channel within its 10-dB bandwidth. Second, for the cumulative crosstalk, within the 10-dB bandwidth of a channel, the power of all other channels is added up. The cumulative crosstalk is the difference of the maximum of this added crosstalk with the peak of the channel [13]. The 10-dB bandwidth is picked as we consider this the relevant operating range of each channel. The conventional and cumulative crosstalk of a device are taken as the maximum of that type of crosstalk of any channel. Both types of crosstalk are illustrated in Fig. 2.

\section{FABRICATION AND DESIGN}

The fabrication of the structures was done by fir $t$ depositing a $220 \mathrm{~nm} \mathrm{Si} \mathrm{N}_{3} \mathrm{~N}_{4}$ layer on a $\mathrm{Si}$ wafer coated with $2.6 \mu \mathrm{m}$ oxide. Structures were etched into the substrate by means of $193 \mathrm{~nm}$ optical lithography. No top cladding was added. This process is back-end CMOS compatible. More information on the fabrication process can be found in [17]. A microscopy image of an AWG is shown in Fig. 3.

This $\mathrm{Si}_{3} \mathrm{~N}_{4}$ material platform has a relatively high-index-contrast, allowing tight bends and therefore

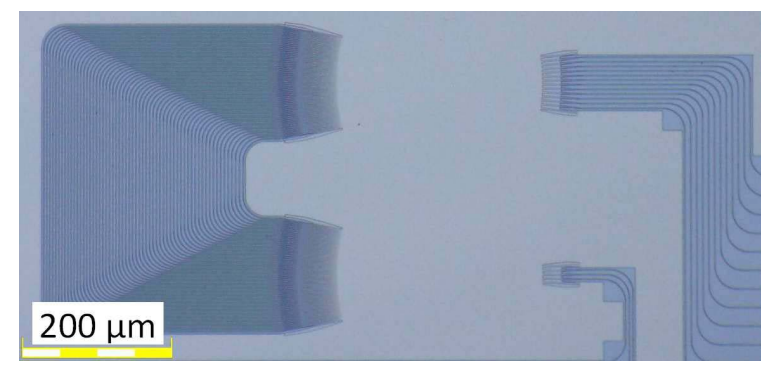

Fig. 3. Microscopy image of $\mathrm{Si}_{3} \mathrm{~N}_{4}$ AWG.

smaller-footprint devices. This HIC unfortunately also brings a high sensitivity to fabrication errors, like sidewall roughness and waveguide width variation. The former predominantly results in high phase errors, increasing crosstalk. A few fabrication and design adaptations were made to alleviate these problems, based on successful results for similar devices in silicon-on-insulator [2], [18].

First, a double etch step is used: in the access waveguides and the bends in the array, a full $220 \mathrm{~nm}$ etch is applied in the cladding region, whereas for the apertures of the star coupler a $70 \mathrm{~nm}$ etch is used in the cladding region to lower the index contrast. Second, straight waveguide sections have a width of $0.9 \mu \mathrm{m}$ to reduce the effect of sidewall roughness, a major contributor to phase errors. In these regions the waveguides can become multimode. This is counteracted, as in bends the waveguide is tapered down to $0.7 \mu \mathrm{m}$, ensuring single-mode behavior. This is shown in Fig. 1(b). Third, upon entering the star coupler, the width of the (shallow etched) aperture is gradually increased to $1.5 \mu \mathrm{m}-2 \mu \mathrm{m}$, reducing ref ections upon transition to the slab region [18]. An AWG aperture is shown in Fig. 1(c) illustrating the shallow etch cladding region as well as the expanding waveguide width.

We designed sets of AWGs with $0.5 \mathrm{~nm}, 1 \mathrm{~nm}$ and $2 \mathrm{~nm}$ target channel spacing, with $1.5,1.8$ or $2.0 \mu \mathrm{m}$ aperture width. All AWGs have 12 channels and 60 delay lines. Grating couplers were implemented for vertical coupling of light to and from the chip [19]. We used the IPKISS design framework to design and simulate the arrayed waveguide grating circuit [20].

\section{Measurement Procedure}

The devices were measured in a fully automated f ber-to$f$ ber setup, where the in- and out-put $f$ bers are aligned to their respective grating couplers with an alignment precision of $0.01 \mu \mathrm{m}$. A tunable laser was used as a light source, and the output was measured with a power meter. For each measurement of an output channel of each AWG, a reference measurement was carried out immediately after, with the f ber position optimized for the peak wavelength of the channel. The reference structure comprised two grating couplers connected by a short straight waveguide. These reference measurements were included to eliminate the effect of the grating couplers. For the data analysis, after normalization by means of the reference measurement, a Gaussian was $\mathrm{ftted}$ to each channel and this Gaussian was used for further characterization of the spectrum in order to reduce the effect of noise. 
TABLE I

Measured Characteristics of Different $\mathrm{Si}_{3} \mathrm{~N}_{4}$ AWGs

\begin{tabular}{|c|c|c|c|}
\hline & AWG 1 & AWG 2 & AWG 3 \\
\hline Average Channel Spacing $(\mathrm{nm})$ & 1.991 & 0.988 & 0.498 \\
\hline Center Wavelength $(\mathrm{nm})$ & 890.6 & 905.41 & 905.38 \\
\hline Insertion Loss $(\mathrm{dB})$ & -1.21 & -2.26 & -3.99 \\
\hline Insertion Loss Non-uniformity(dB) & -1.28 & -0.85 & -0.96 \\
\hline Conventional Crosstalk(dB) & 20.1 & 19.5 & 8.2 \\
\hline Cumulative Crosstalk(dB) & 16.9 & 14.7 & 4.2 \\
\hline
\end{tabular}

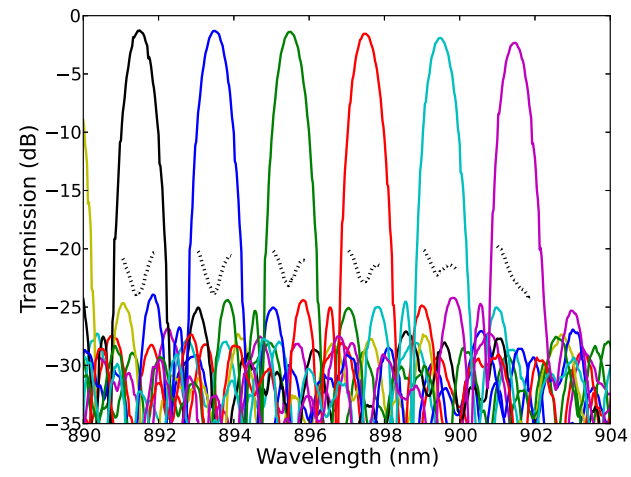

(a)

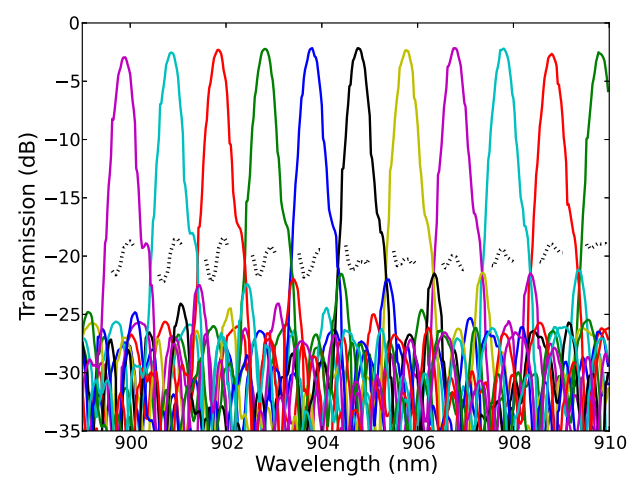

(b)

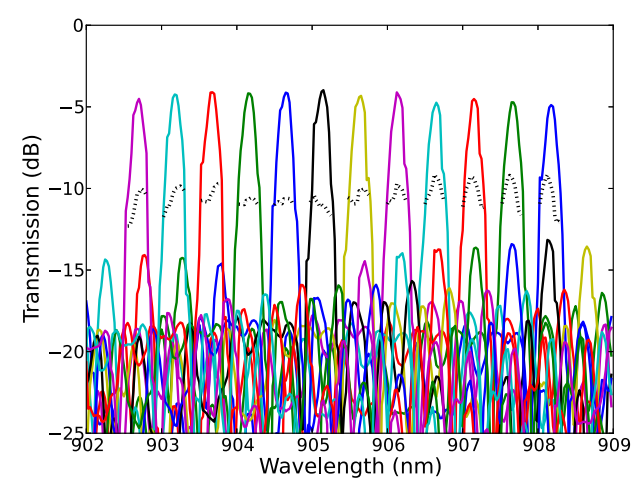

(c)

Fig. 4. Experimental AWG spectra with different design channel spacings. The dotted lines are the cumulative crosstalk. (a) $2 \mathrm{~nm}$ design channel spacing. (b) $1 \mathrm{~nm}$ design channel spacing. (c) $0.5 \mathrm{~nm}$ design channel spacing.

\section{EXPERIMENTAL RESULTS}

Figures 4(a), 4(b) and 4(c) show the measured spectral responses for AWGs with channel spacing of 2, 1 and $0.5 \mathrm{~nm}$ respectively. Unfortunately, for the former two devices,
TABLE II

COMPARISON OF ARRAYED WAVEguide GRATINGS OPERATING IN THE 600-900 nm WAVELENGTH RANGE

\begin{tabular}{|c||c|c|c|c|c|}
\hline Material & SiON [9] & PDMS [10] & \multicolumn{2}{|c|}{ Silica } & \multirow{2}{*}{$\mathrm{Si}_{3} \mathrm{~N}_{4}$} \\
\cline { 4 - 5 } & $(1.51)$ & $(1.429)$ & {$[8]$} & {$[11]$} & (this work)(1.9) \\
\hline $\begin{array}{c}\text { Central } \\
\text { Wavelength (nm) }\end{array}$ & 881 & 641.5 & 699 & 800 & 892 \\
\hline $\begin{array}{c}\text { Channel } \\
\text { Spacing (nm) }\end{array}$ & 5.5 & 1 & 0.75 & 12.5 & 2 \\
\hline No. channels & 41 & 4 & 8 & 8 & 12 \\
\hline $\begin{array}{c}\text { Insertion } \\
\text { Loss (dB) }\end{array}$ & $-1.1 \pm 2$ & -11.4 & -4.5 & -5 & -1.2 \\
\hline Crosstalk (dB) & - & 16 & $>25$ & $>20$ & 20 \\
\hline Footprint (mm $\left.{ }^{2}\right)$ & 340 & 112 & 240 & 130 & 0.34 \\
\hline
\end{tabular}

we were unable to measure a full free spectral range due to the tunable laser, which was limited in wavelength range to $890-910 \mathrm{~nm}$. The obtained data did suff ce to perform an analysis and obtain the parameters of interest. The results of the analysis of the measured data are given in Table I.

For all devices, the experimental channel spacing is in very good agreement with its design value. The most noteworthy feature of these devices is their low insertion loss, especially considering their small footprint. Comparing the structures, those with a smaller channel spacing have a larger crosstalk, and to a lesser extent a larger insertion loss. This is presumably because devices with smaller channel spacing require a larger $\Delta \mathrm{L}$, and hence have a larger footprint and therefore suffer more from phase errors, reducing their performance, particularly in terms of crosstalk.

Due to this trend, the device with $2 \mathrm{~nm}$ channel spacing, for which the spectrum is plotted in Fig. 4, has the lowest insertion loss, only $-1.21 \mathrm{~dB}$. It exhibits conventional and cumulative crosstalk of $20 \mathrm{~dB}$ and $16.9 \mathrm{~dB}$, respectively. The footprint of the device is only $0.45 \times 0.75 \mathrm{~mm}^{2}$. The insertion loss non-uniformity of the device is $-1.3 \mathrm{~dB}$ and its $3-\mathrm{dB}$ bandwidth is $0.59 \mathrm{~nm}$.

In Table II a comparison of this device with the stateof-the-art AWGs in the $600-900 \mathrm{~nm}$ wavelength range is given. When multiple devices were available, the one with central wavelength and channel spacing closest to our own was preferred. Our device shows great performance in terms of insertion loss and crosstalk but especially excels due its small footprint, two orders of magnitude smaller than the other devices.

\section{CONCLUSION}

We report state-of-the-art arrayed waveguide gratings in the high-index silicon nitride material platform for the $900 \mathrm{~nm}$ wavelength range. Due to design adaptions, a low insertion loss and low crosstalk are achieved, despite the HIC platform. The footprint of these devices is much smaller than that of the other AWGs reported so far for VNIR wavelength. The reported devices are furthermore fully CMOS-compatible, and therefore suited for cheap large-scale production. Due to the combination of these merits, these AWGs pave the way for a wide range of applications, in particular on-chip photonic sensing. 


\section{ACKNOWLEDGMENT}

The authors acknowledge imec Leuven for $\mathrm{Si}_{3} \mathrm{~N}_{4}$ processing.

\section{REFERENCES}

[1] M. K. Smit and C. Van Dam, "PHASAR-based WDM-devices: Principles, design and applications," IEEE J. Sel. Topics Quantum Electron., vol. 2, no. 2, pp. 236-250, Jun. 1996.

[2] W. Bogaerts et al., "Silicon-on-insulator spectral f lters fabricated with CMOS technology," IEEE J. Sel. Topics Quantum Electron., vol. 16, no. 1, pp. 33-44, Jan./Feb. 2010.

[3] E. Ryckeboer et al., "Silicon-on-insulator spectrometers with integrated GaInAsSb photodiodes for wide-band spectroscopy from 1510 to 2300 nm," Opt. Exp., vol. 21, no. 5, pp. 6101-6108, 2013.

[4] T. Claes, W. Bogaerts, and P. Bienstman, "Vernier-cascade label-free biosensor with integrated arrayed waveguide grating for wavelength interrogation with low-cost broadband source," Opt. Lett., vol. 36, no. 17, pp. 3320-3322, 2011

[5] A. Malik et al., "Germanium-on-silicon mid-infrared arrayed waveguide grating multiplexers," IEEE Photon. Technol. Lett., vol. 25, no. 18, pp. 1805-1808, Sep. 15, 2013.

[6] M. Muneeb et al., "Demonstration of silicon-on-insulator mid-infrared spectrometers operating at $3.8 \mu \mathrm{m}, "$ Opt. Exp., vol. 21, no. 10, pp. 11659-11669, 2013.

[7] S. Pathak, D. Van Thourhout, and W. Bogaerts, "Design trade-offs for silicon-on-insulator-based AWGs for (de)multiplexer applications," Opt. Lett., vol. 38, no. 17, pp. 2961-2964, 2013.

[8] K. Suzuki, Y. Hida, T. Shibata, Y. Inoue, H. Takahashi, and K. Okamoto, "Silica based arrayed waveguide gratings for the visible wavelength range," NTT Tech. Rev., vol. 4, no. 6, pp. 48-52, 2006.

[9] N. Ismail et al., "Improved arrayed-waveguide-grating layout avoiding systematic phase errors," Opt. Exp., vol. 19, no. 9, pp. 8781-8794, 2011.

[10] J. S. Kee, D. P. Poenar, P. Neužil, L. Yobaş, and Y. Chen, "Design and fabrication of poly(dimethylsiloxane) arrayed waveguide grating," Opt. Exp., vol. 18, no. 21, pp. 21732-21742, 2010.
[11] Y. Komai, H. Nagano, K. Okamoto, and K. Kodate, "Compact spectroscopic sensor using a visible arrayed waveguide grating," Jpn. J. Appl. Phys., vol. 45, no. 8S, p. 6742, 2006.

[12] Y. Oikawa, H. Hasegawa, K. Suzuki, Y. Inoue, T. Hirooka, and M. Nakazawa, " $4 \times 10 \mathrm{~Gb} / \mathrm{s}$ WDM transmission over a 5-km-long photonic crystal fibe in the $800-\mathrm{nm}$ region," IEEE Photon. Technol. Lett., vol. 19, no. 8, pp. 613-615, Apr. 15, 2007.

[13] S. Pathak et al., "Effect of mask discretization on performance of silicon arrayed waveguide gratings," IEEE Photon. Technol. Lett., vol. 26, no. 7, pp. 718-721, Apr. 1, 2014.

[14] M. Piels, J. F. Bauters, M. L. Davenport, M. J. R. Heck, and J. E. Bowers, "Low-loss silicon nitride AWG demultiplexer heterogeneously integrated with hybrid III-V/silicon photodetectors," J. Lightw. Technol., vol. 32, no. 4, pp. 817-823, Feb. 15, 2014.

[15] D. Dai et al., "Low-loss $\mathrm{Si}_{3} \mathrm{~N}_{4}$ arrayed-waveguide grating (de)multiplexer using nano-core optical waveguides," Opt. Exp., vol. 19, no. 15, pp. 14130-14136, 2011.

[16] L. Chen, C. R. Doerr, L. Buhl, Y. Baeyens, and R. A. Aroca, "Monolithically integrated 40-wavelength demultiplexer and photodetector array on silicon," IEEE Photon. Technol. Lett., vol. 23, no. 13, pp. 869-871, Jul. 1, 2011.

[17] A. Z. Subramanian et al., "Low-loss singlemode PECVD silicon nitride photonic wire waveguides for 532-900 nm wavelength window fabricated within a CMOS pilot line," IEEE Photon. J., vol. 5, no. 6, Dec. 2013, Art. ID 2202809.

[18] S. Pathak, M. Vanslembrouck, P. Dumon, D. Van Thourhout, and W. Bogaerts, "Compact SOI-based polarization diversity wavelength de-multiplexer circuit using two symmetric AWGs," Opt. Exp., vol. 20, no. 26, pp. B493-B500, 2012.

[19] A. Z. Subramanian, S. Selvaraja, P. Verheyen, A. Dhakal, K. Komorowska, and R. Baets, "Near-infrared grating couplers for silicon nitride photonic wires," IEEE Photon. Technol. Lett., vol. 24, no. 19 , pp. 1700-1703, Oct. 1, 2012.

[20] W. Bogaerts et al., "Integrated design for integrated photonics: From the physical to the circuit level and back," Proc. SPIE, vol. 8781, p. 878102 , May 2013. 